Pacific Journal of Mathematic 


\section{CONVOLUTION MULTIPLIERS AND DISTRIBUTIONS}

\section{ÁrPÁD SzÁZ}

In this paper, in a purely algebraic way, Schwartz distributions in several variables are generalized in accordance with their homomorphism interpretation proposed by $\mathbf{R}$. A. Struble.

0. Introduction. R. A. Struble in [10] has shown that Schwartz distributions can be characterized simply as mappings, from the space $\mathscr{D}$ of test functions into the space $\mathscr{E}$ of smooth functions, which commute with ordinary convolution. This new view of distributions has turned out to be very useful $[11,12]$ and motivated us to give a simple generalization for distributions which is closely related to Mikusiński operators and convolution quotients of other types [11, 12, $4,13]$. The method employed here is an appropriate modification of a general algebraic method $[5,2,8]$.

Mappings which commute with convolution are called convolution multipliers here. (Distributions can be characterized as convolution multipliers, Mikusiński operators themselves are convolution multipliers.)

In $\S 1$, convolution multipliers from various subsets of $\mathscr{D}$ into $\mathscr{E}$ are discussed. We are primarily concerned with their maximal extensions.

In $\$ 2$, a module $\mathfrak{M}$ of certain maximal convolution multipliers is constructed and investigated from an algebraic point of view.

In $\S 3$, Schwartz distributions are embedded and characterized in $\mathfrak{M}$. For example, we prove that distributions are the only continuous elements of $\mathfrak{M}$. Finally, we show that there are elements in $\mathfrak{M}$ which are not distributions.

To illustrate the appropriateness of our generalizations, we refer to the following facts:

One of the difficulties in working with Schwartz distributions is that only distributions $\Lambda$ satisfying $\Lambda * \mathscr{D}=\mathscr{D}$ are invertible in $\mathscr{D}^{\prime}$. Whereas, distibutions $\Lambda$ satisfying $\Lambda * \mathscr{D} \subset \mathscr{D}$ such that $\Lambda * \mathscr{D}$ has no proper annihilators in $\mathscr{E}$ are invertible in $\mathfrak{M}$. (The heat operator in two dimensions [1] seems to be a distribution which is not invertible in $\mathscr{D}^{\prime}$, but is invertible in $\mathfrak{M}$.)

There are regular Mikusiński operators [1] which are not distributions. Whereas, normal Mikusiński operators [11] can be embedded in $\mathfrak{M}$.

1. Convolution multipliers and their maximal extensions. Let $k$ be a fixed positive integer, $\mathbf{R}^{k}$ be the $k$ dimensional Euclidean space and $\mathbf{C}$ be the field of complex numbers. 
Let $\mathscr{E}$ be the set of all infinitely differentiable functions from $\mathbf{R}^{k}$ into $\mathbf{C}$, and let $\mathscr{D}$ be the subset of $\mathscr{E}$ consisting of all those functions with compact support.

It is known that with the pointwise linear operations and convolution $\mathscr{D}$ is a commutative complex algebra without proper zero-divisors, and $\mathscr{E}$ is a complex vector space and a $\mathscr{D}$-module.

DEFINITION 1.1. Let

$$
M=\left\{F: D_{F} \subset \mathscr{D} \rightarrow \mathscr{E}: \forall \varphi, \psi \in D_{F}: F(\varphi) * \psi=\varphi * F(\psi)\right\}
$$

Proposition 1.2. Let $F \in M$. Then $F$ has a maximal extension in M.

Proof. Let $\mathscr{F}=\{G \in M: F \subset G\}$. Under set inclusion $\mathscr{F}$ is a novoid partially ordered set. Moreover, if $\mathscr{B}$ is a chain in $\mathscr{F}$, then $\cup \mathscr{B}$ is an upper bound for it in $\mathscr{F}$. (To prove this use (2.19) Theorem in [3].) Thus, by Zorn's lemma $\mathscr{F}$ has a maximal member.

Definition 1.3. Let $D \subset \mathscr{D}$. Then $D$ is said to be normal [11] if and only if $D \neq \phi$ and $D$ has no proper annihilators in $\mathscr{E}$, i.e., $f \in \mathscr{E}$ and $f * D=\{0\}$ imply that $f=0$.

LEMma 1.4. Let $D \subset \mathscr{D}$. Suppose that for every $0<\epsilon \in \mathbf{R}^{1}$ there exists $\varphi \in \mathscr{D}$ such that $0 \leqq \varphi$ or $\varphi \leqq 0$ and the diameter of the support of $\varphi$ is less than $\epsilon$. Then $D$ is normal. In particular, $\mathscr{D}$ is normal.

Proof. See the proof of 1.5. Lemma in [4].

Proposition 1.5. Let $D \subset \mathscr{D}$. Then every $F \in M$ with domain $D$ has a unique maximal extension in $M$ if and only if $D$ is normal.

Proof. Suppose first that every $F \in M$ with domain $D$ has a unique maximal extension in $M$. To prove that $D$ is normal suppose that $f \in \mathscr{E}$ such that $f * D=\{0\}$. Let $F$ and 0 be the functions defined on $\mathscr{D}$ by $F(\varphi)=f * \varphi$ and $O(\varphi)=0$. Then $F$ and 0 are maximal extensions of $F \mid D$ in $M$. Thus, $f * \varphi=0$ for all $\varphi \in \mathscr{D}$. Hence, it follows that $f=0$.

To prove the converse suppose that $D$ is normal and $F \in M$ with domain $D$. We prove that

$$
\bar{F}=\{(\varphi, f) \in \mathscr{D} \times \mathscr{E}: \forall \sigma \in D: f * \sigma=\varphi * F(\sigma)\}
$$

is the unique maximal extension of $F$ in $M$. 
To prove that $\bar{F}$ is a function, suppose that $\left(\varphi, f_{1}\right),\left(\varphi, f_{2}\right) \in$ $\bar{F}$. Then

$$
f_{1} * \sigma=\varphi * F(\sigma) \text { and } f_{2} * \sigma=\varphi * F(\sigma)
$$

for all $\sigma \in D$. Hence, it follows that $\left(f_{1}-f_{2}\right) * \sigma=0$ for all $\sigma \in D$. This implies that $f_{1}=f_{2}$.

Now, we prove that $\bar{F}$ is a multiplier. For this, suppose that $\varphi, \psi \in D_{\bar{F} .} \quad$ Then

$$
\bar{F}(\varphi) * \sigma=\varphi * F(\sigma) \text { and } \bar{F}(\psi) * \sigma=\psi * F(\sigma)
$$

for all $\sigma \in D$. Hence, it follows that

$$
(\bar{F}(\varphi) * \psi-\varphi * \bar{F}(\psi)) * \sigma=0
$$

for all $\sigma \in D$. This implies that $\bar{F}(\varphi) * \psi=\varphi * \bar{F}(\psi)$.

The remaining part of the proof is quite obvious.

REMARK 1.6. In the following, we shall not make use of Proposition 1.2. and 1.5. They are only to make clear and complete our treatment.

DEFINITION 1.7. Let

$$
\mathcal{M}=\left\{F \in M: D_{F} \text { is normal }\right\}
$$

For any $F \in \mathcal{M}$, let

$$
\bar{F}=\left\{(\varphi, f) \in \mathscr{D} \times \mathscr{E}: \forall \sigma \in D_{\mathrm{F}}: f * \sigma=\varphi * F(\sigma)\right\} .
$$

THEOREM 1.8. Let $F \in M$. Then $\bar{F}$ is the unique maximal extension of $F$ in $M$.

Proof. See the second part of the proof of Proposition 1.5.

Corollary 1.9. Let $F \in M$. Then $D_{\bar{F}}$ is an algebra ideal in $\mathscr{D}$, and $\bar{F}$ is a vector space and a $\mathscr{D}$-module homomorphism.

Proof. Easy computation.

Corollary 1.10. Let $F, G \in M$. Suppose that $D \subset D_{F} \cap D_{G}$ such that $D$ is normal and $F(\varphi)=G(\varphi)$ for all $\varphi \in D$. Then $\bar{F}=\bar{G}$.

Proof. Trivial. 


\section{A module of convolution multipliers.}

DEFINITION 2.1. Let

$$
\mathfrak{M}=\{\bar{F}: F \in \mathcal{M}\}
$$

and

$$
\mathfrak{R}=\left\{\bar{\Phi}: \Phi \in \mathcal{M}, \Phi\left(D_{\Phi}\right) \subset \mathscr{D}\right\}
$$

For any $F, G \in \mathfrak{M}$ and $\Phi \in \mathfrak{N}$, let

$$
F+G=\overline{F+G}
$$

and

$$
\Phi * F=F * \Phi=\overline{F \circ \Phi} .
$$

THEOREM 2.2. With + and $*, \mathfrak{N}$ is a commutative ring with unity and without proper zero-divisors, and $\mathfrak{M}$ is a unitial $\mathfrak{N}$-module.

Proof. In the course of this proof we shall often use the following obvious fact: If $D_{1}, D_{2} \subset \mathscr{D}$ are normal then $D_{1} * D_{2}$ is normal. Moreover, if in addition $D_{1} * \mathscr{D} \subset D_{1}$ and $D_{2} * \mathscr{D} \subset D_{2}$, then $D_{1} * D_{2} \subset D_{1} \cap D_{2}$, and so $D_{1} \cap D_{2}$ is also normal.

Suppose that $F, G \in \mathfrak{M}$ and $\Phi, \Psi \in \mathfrak{N}$. From the definition of $\mathfrak{M}$ it follows immediately that $\Phi^{-1}(\mathscr{D})$ and $\Psi^{-1}(\mathscr{D})$ are normal algebra ideals in D.

The first step is to prove that $f+G, F * \Phi \in \mathfrak{M}$ and $\Phi+\Psi, \Phi * \Psi \in$ $\mathfrak{N}$. For example, we prove that $F * \Phi \in \mathfrak{M}$. For all $\varphi \in D_{F}$ and $\psi \in \Phi^{-1}(\mathscr{D})$ we have

$$
(F \circ \Phi)(\varphi * \psi)=F(\Phi(\varphi * \psi))=F(\varphi * \Phi(\psi))=F(\varphi) * \Phi(\psi) .
$$

Hence, it follows that $D_{F} * \Phi^{-1}(\mathscr{D}) \subset D_{F \circ \Phi}$. Thus $D_{\text {Fo }}$ is normal. Moreover, for all $\varphi, \psi \in D_{F \circ \Phi}$, we have

$$
\begin{aligned}
(F \circ \Phi)(\varphi) * \psi & =F(\Phi(\varphi)) * \psi=F(\Phi(\varphi) * \psi)=F(\varphi * \Phi(\psi)) \\
& =\varphi * F(\Phi(\psi))=\varphi *(F \circ \Phi)(\psi) .
\end{aligned}
$$

Thus $F \circ \Phi \in M$, and so $F * \Phi=\overline{F \circ \Phi} \in \mathfrak{M}$.

The next step is to prove the required commutative, associative and distributive laws for + and $*$. For example, we prove that $F *(\Phi+$ $\Psi)=F * \Phi+F * \Psi$. Clearly, $D_{F} *\left(\Phi^{-1}(\mathscr{D}) \cap \Psi^{-1}(\mathscr{D})\right)$ is normal and for all $\varphi \in D_{F} *\left(\Phi^{-1}(\mathscr{D}) \cap \Psi^{-1}(\mathscr{D})\right)$, we have 


$$
\begin{aligned}
(F *(\Phi+\Psi))(\varphi) & =(F \circ(\Phi+\Psi))(\varphi)=F(\Phi(\varphi)+\Psi(\varphi)) \\
& =F(\Phi(\varphi))+F(\Psi(\varphi)) \\
& =(F \circ \Phi+F \circ \Psi)(\varphi)=(F * \Phi+F * \Psi)(\varphi) .
\end{aligned}
$$

Hence, using Corollary 1.10, we get $F *(\Phi+\Psi)=F * \Phi+F * \Psi$.

Now, let 0 and 1 be the functions defined on $\mathscr{D}$ by

$$
0(\varphi)=0 \quad \text { and } \quad 1(\varphi)=\varphi
$$

Then $0,1 \in \mathfrak{N}$ and

$$
\begin{gathered}
F+0=\overline{F+0}=\bar{F}=F, \\
F+(-F)=\overline{F+(-F)}=\overline{0 \mid D_{F}}=0 \\
F * 1=\overline{F \circ 1}=\bar{F}=F .
\end{gathered}
$$

Finally, we prove that $\mathfrak{N}$ has no proper zero-divisors. For this suppose that $\Phi * \Psi=0$. Then for all $\varphi \in \Phi^{-1}(\mathscr{D})$ and $\psi \in \Psi^{-1}(\mathscr{D})$, we have

$$
(\Phi * \Psi)(\varphi * \psi)=\Phi(\varphi) * \Psi(\psi)=0
$$

Since $\mathfrak{N}$ is commutative, it is no restriction to assume that $\Psi \neq 0$. Then there exists $\psi_{0} \in \Psi^{-1}(\mathscr{D})$ such that $\Psi\left(\psi_{0}\right) \neq 0$. Moreover, for all $\varphi \in$ $\Phi^{-1}(\mathscr{D})$, we have $\Phi(\varphi) * \Psi\left(\psi_{0}\right)=0$. Thus $\Phi(\varphi)=0$ for all $\varphi \in \Phi^{-1}(\mathscr{D})$, and so $\Phi=0$.

Lemma 2.3. (i) Let $F \in \mathfrak{M}$. Then $F$ is injective if and only if the range of $F$ has no proper annihilators in $\mathscr{D}$.

(ii) Let $\Phi \in \mathfrak{N}$. Then $\Phi$ is injective if and only if $\Phi \neq 0$.

Proof. (i) Suppose first that $F$ is injective. Let $0 \neq \varphi \in D_{F}$, $\chi \in \mathscr{D}$ and suppose that $\chi * F\left(D_{F}\right)=\{0\}$. Then

$$
F(\chi * \varphi)=\chi * F(\varphi)=0 .
$$

Hence, since $F$ is injective and $F(0)=0$, it follows that $\chi * \varphi=0$. This implies that $\chi=0$.

Suppose now that $F\left(D_{F}\right)$ has no proper annihilators in $\mathscr{D}$. Let $\varphi_{1}, \varphi_{2} \in D_{F}$ and suppose that $F\left(\varphi_{1}\right)=F\left(\varphi_{2}\right)$. Then

$$
\varphi_{1} * F(\psi)=F\left(\varphi_{1}\right) * \psi=F\left(\varphi_{2}\right) * \psi=\varphi_{2} * F(\psi)
$$

i.e., $\left(\varphi_{1}-\varphi_{2}\right) * F(\psi)=0$ for all $\psi \in D_{F}$. Hence, by the assumption, it follows that $\varphi_{1}=\varphi_{2}$. 
(ii) The necessity is trivial. Suppose now that $\Phi \neq 0$. Then there exists $\varphi \in \Phi^{-1}(\mathscr{D})$ such that $\Phi(\varphi) \neq 0$. This implies that $\Phi\left(D_{\Phi}\right)$ has no proper annihilators in $\mathscr{D}$.

Theorem 2.4. Let $\Phi \in \mathfrak{N}$. Then $\Phi$ is invertible in $\mathfrak{N}$ if and only if the range of $\Phi$ has normal intersection with $\mathscr{D}$.

Proof. Suppose first that $\Phi$ is invertible in $\mathfrak{N}$. Then there exists $\Psi \in \mathfrak{N}$ such that $\Phi * \Psi=1$. Thus, for all $\varphi \in D_{\Phi} * \Psi^{-1}(D)$, we have

$$
(\Phi * \Psi)(\varphi)=\Phi(\Psi(\varphi))=1(\varphi)=\varphi
$$

Hence $D_{\Phi} * \Psi^{-1}(\mathscr{D}) \subset \Phi\left(D_{\Phi}\right) \cap \mathscr{D}$. Thus $\Phi\left(D_{\Phi}\right) \cap \mathscr{D}$ is normal.

Suppose now that $\Phi\left(D_{\Phi}\right) \cap \mathscr{D}$ is normal. Then $\Phi \neq 0$, and so $\Phi$ is injective. Thus $\Phi^{-1}$ is a function. Moreover, for any $\varphi, \psi \in \Phi\left(D_{\Phi}\right) \cap$ $\mathscr{D}$ we have

$$
\Phi\left(\Phi^{-1}(\varphi) * \psi\right)=\Phi\left(\Phi^{-1}(\varphi)\right) * \psi=\varphi * \psi
$$

and

$$
\Phi\left(\varphi * \Phi^{-1}(\psi)\right)=\varphi * \Phi\left(\Phi^{-1}(\psi)\right)=\varphi * \psi
$$

i.e., $\Phi^{-1}(\varphi) * \psi=\varphi * \Phi^{-1}(\psi)$. Thus $\Phi^{-1} \mid \Phi\left(D_{\Phi}\right) \cap \mathscr{D} \in \mathcal{M}$. We prove

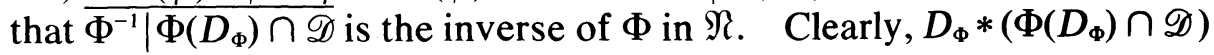
is normal, and for all $\varphi \in D_{\Phi} *\left(\Phi\left(D_{\Phi}\right) \cap \mathscr{D}\right)$ we have

$$
\left(\Phi * \overline{\left.\Phi^{-1} \mid \Phi\left(D_{\Phi}\right) \cap \mathscr{D}\right)}(\varphi)=\Phi\left(\Phi^{-1}(\varphi)\right)=\varphi=1(\varphi) .\right.
$$

This implies that $\Phi * \overline{\Phi^{-1} \mid \Phi\left(D_{\Phi}\right) \cap \mathscr{D}}=1$.

EXAmPLES 2.5. Let $0 \neq \varphi \in \mathscr{D}$ such that $\int_{\mathbf{R}^{k}} \varphi=0$, and let $\Phi$ be the function defined on $\mathscr{D}$ by $\Phi(\psi)=\varphi * \psi$. Then $0 \neq \Phi \in \Re$, but $\Phi$ is not invertible in $\mathfrak{R}$.

Namely, $\Phi\left(D_{\Phi}\right) \cap \mathscr{D}=\varphi * \mathscr{D}$ is not normal, since $f *(\varphi * \mathscr{D})=\{0\}$ for all constant $f \in \mathscr{E}$.

3. Embedding of distributions. Let $\mathscr{D}^{\prime}$ be the set of all Schwartz distributions on $\mathbf{R}^{k}$, and let $\mathscr{E}^{\prime}$ be the subset of $\mathscr{D}^{\prime}$ consisting of all those distributions with compact support.

It is known that, under addition and convolution, $\mathscr{E}^{\prime}$ is a commutative ring with unity and without proper zero-divisors, and $\mathscr{D}^{\prime}$ is a unitial $\mathscr{E}^{\prime}$-module. Moreover, $\mathbf{C}$ and $\mathscr{E}$ are embedded in $\mathscr{D}^{\prime}$ such that C, $\mathscr{D} \subset \mathscr{E}^{\prime}$. 
Definition 3.1. For any $\Lambda \in \mathscr{D}^{\prime}$, let $F_{\Lambda}$ be the function defined on $\mathscr{D}$ by

$$
F_{\Lambda}(\varphi)=\Lambda * \varphi
$$

THEOREM 3.2. The mapping defined on $\mathscr{D}^{\prime}$ by

$$
\Lambda \rightarrow F_{\Lambda}
$$

is an injection from $\mathscr{D}^{\prime}$ into $\mathfrak{M}$ taking $\mathscr{E}$ ' into $\mathfrak{M}$ such that

$$
F_{\Lambda_{1}+\Lambda_{2}}=F_{\Lambda_{1}}+F_{\Lambda_{2}} \text { for all } \Lambda_{1}, \Lambda_{2} \in \mathscr{D}^{\prime}
$$

and

$$
F_{\Lambda_{1} * \Lambda_{2}}=F_{\Lambda_{1}} * F_{\Lambda_{2}} \text { for all } \Lambda_{1} \in \mathscr{E}^{\prime} \text { and } \Lambda_{2} \in \mathscr{D}^{\prime}
$$

Proof. Easy computation.

REMARK 3.3. Theorem 3.2. allows us to more and less identify $\Lambda$ with $F_{\Lambda}$. But, for the easier understanding, we shall not make this identification complete.

Theorem 3.4. Let $F \in \mathfrak{M}$. Then $F=F_{\Lambda}$ for some $\Lambda \in \mathscr{D}^{\prime}$ if and only if $D_{F}=\mathscr{D}$.

Proof. See Lemma 3 in [10].

In the following theorem $\mathscr{E}$ and $\mathscr{D}$ are supposed to be equipped with their usual topologies [9].

Theorem 3.5. Let $F \in \mathfrak{M}$. Then $F=F_{\Lambda}$ for some $\Lambda \in \mathscr{D}^{\prime}$ if and only if $F$ is continuous.

Proof. Suppose first that $F=F_{\Lambda}$ for some $\Lambda \in \mathscr{D}^{\prime}$. Then $F(\varphi)=$ $\Lambda * \varphi$ for all $\varphi \in \mathscr{D}$. Thus, by 6.33 Theorem in [9], $F$ is continuous.

Suppose now that $F$ is continuous. Let $\lambda$ be the function defined on $\check{D}_{F}=\left\{\check{\varphi}: \varphi \in D_{F}\right\}$ by

$$
\lambda(\varphi)=F(\check{\varphi})(0)
$$

Then $\lambda$ is a continuous linear functional on the linear subspace $\check{D}_{F}$ of $\mathscr{D}$. Thus, by 3.6 Theorem in [9], there exists $\Lambda \in \mathscr{D}^{\prime}$ such that $\lambda \subset$ $\Lambda$. Moreover, we have 


$$
\begin{aligned}
F_{\Lambda}(\varphi)(t) & =(\Lambda * \varphi)(t)=\Lambda\left(\mathscr{T}_{t} \check{\varphi}\right)=\lambda\left(\mathscr{T}_{t} \check{\varphi}\right)=F\left(\left(\mathscr{T}_{t} \check{\varphi}\right)^{\vee}\right)(0) \\
& =F\left(\mathscr{T}_{-t} \varphi\right)(0)=\left(\mathscr{T}_{-t} F(\varphi)\right)(0)=F(\varphi)(t)
\end{aligned}
$$

for all $\varphi \in D_{F}$ and $t \in \mathbf{R}^{k}$. Hence, it follows that $F=F_{\Lambda}$.

REMARK 3.6. The proof of Theorem 3.5 shows that we might have used an apparently weaker continuity property of $F$ in Theorem 3.5., if we had taken the topology of the pointwise convergence on $\mathbf{R}^{k}$ instead of the usual topology of $\mathscr{E}[12]$.

LEMma 3.7. Let $\Phi \in \Re$. Suppose that $(\sigma)_{n=1}^{\infty}$ is an approximate identity (delta sequence [12]) such that $\left(\sigma_{n}\right)_{n=1}^{\infty} \subset D_{\Phi}$ and $\left\{\Phi\left(\sigma_{n}\right)\right\}_{n=1}^{\infty} \subset \mathscr{D}$. Then $\Phi\left(D_{\Phi}\right) \subset \mathscr{D}$.

Proof. Let $\varphi, \psi \in D_{\Phi}$ such that $\Phi(\psi) \in \mathscr{D}$. Then

$$
\Phi(\varphi) * \sigma_{n}=\varphi * \Phi\left(\sigma_{n}\right) \text { and } \Phi\left(\sigma_{n}\right) * \psi=\sigma_{n} * \Phi(\psi)
$$

for all $n$. Hence,

$$
\operatorname{supp} \Phi(\varphi) * \sigma_{n} \subset \operatorname{supp} \varphi+\operatorname{supp} \Phi\left(\sigma_{n}\right)
$$

and, using Lions' theorem [12],

$$
\left[\operatorname{supp} \Phi\left(\sigma_{n}\right)\right]+[\operatorname{supp} \psi]=\left[\operatorname{supp} \sigma_{n}\right]+[\operatorname{supp} \Phi(\psi)]
$$

for all $n$. Thus, we have

$$
\operatorname{supp} \Phi(\varphi) * \sigma_{n} \subset \operatorname{supp} \varphi+[\operatorname{supp} \Phi(\psi)]-[\operatorname{supp} \psi]+\left[\operatorname{supp} \sigma_{n}\right]
$$

for all $n$. Therefore, there exists a bounded set $B \subset \mathbf{R}^{k}$ such that

$$
\operatorname{supp} \Phi(\varphi) * \sigma_{n} \subset B
$$

for all $n$. Hence, since $\lim _{n \rightarrow \infty} \Phi(\varphi) * \sigma_{n}=\Phi(\varphi)$, it follows that

$$
\operatorname{supp} \Phi(\varphi) \subset B
$$

EXAmPle 3.8. Let $Q$ be the quotient field of $\mathscr{D}$. Suppose that $q \in Q$ such that $D_{q}$ contains an approximate identity, but $D_{q} \neq \mathscr{D} \backslash\{0\}$. (To make it clear, recall that $Q$ consists of all

$$
\frac{\varphi}{\psi}=\{(\sigma, \chi) \in(\mathscr{D} \mid\{0\}) \times \mathscr{D}: \chi * \psi=\sigma * \varphi\}
$$


such that $\varphi, \psi \in \mathscr{D}$ and $\psi \neq 0$. Concerning the existence of such a $q$ see [1].) Then, it is clear that $q \in \mathcal{M}$, and so $\bar{q} \in \mathfrak{M}$. Moreover, by Lemma 3.7., we have $\bar{q}\left(D_{\bar{q}}\right) \subset \mathscr{D}$. Therefore $\bar{q}=q \cup\{(0,0)\}$. Hence, since $D_{q} \neq \mathscr{D} \backslash\{0\}$, it follows that $D_{\bar{q}} \neq \mathscr{D}$. Thus, by Theorem 3.4., there is no $\Lambda \in \mathscr{D}^{\prime}$ such that $\bar{q}=F_{\Lambda}$.

ACKNOWLEDGEMENT. The author is indebted to J. Erdös for pointing out reference [2] and for his other valuable pieces of advice.

\section{REFERENCES}

1. T. K. Boehme, The support of Mikusiński operators, Trans. Amer. Math. Soc., 176 (1973), 319-334.

2. G. D. Findlay and J. Lambek, A generalized ring of quotients I, II, Canad. Math. Bull., 1 (1958), $77-85 ; 155-167$.

3. E. Hewitt and K. Stromberg, Real and Abstract Analysis, Springer-Verlag, Berlin, 1969.

4. C. C. Hughes, Algebraic operational calculus for Schwartz distributions and Mikusiński operators, Ph. D. Dissertation, North Caroline State University at Raleigh, 1973.

5. R. E. Johnson, The extended centralizer of a ring over a module, Proc. Amer. Math. Soc., 2 (1951), 891-895.

6. R. Larsen, An Introduction to the Theory of Multipliers, Springer-Verlag, Berlin, 1971.

7. M. D. Larsen and P. J. McCharty, Multiplicative Theory of Ideals, Academic Press, London, 1971.

8. L. Máté, Multiplier operators and quotient algebra, Bull. Acad. Polon. Sci. Ser. Sci. Math. Astr. Phys., 8 (1965), 523-526.

9. W. Rudin, Functional Analysis, Mc. Graw-Hill, New York, 1973.

10. R. A. Struble, An algebraic view of distributions and operators, Studia Math., 37 (1971), 103-109.

11. Operator homomorphisms, Math. Z., 130 (1973), 275-285.

12. R. A. Struble and C. C. Hughes, Neocontinuous Mikusiński operators, Trans. Amer. Math. Soc., 185 (1973), 383-400.

13. A. Száz, Convolution quotients and distributions, to appear in Publ. Math. Debrecen.

Received August 12, 1974.

UNIVERSITY OF DEBRECEN, HungaRy 



\section{PACIFIC JOURNAL OF MATHEMATICS}

\section{EDITORS}

RICHARD ARENS (Managing Editor)

University of California

Los Angeles, California 90024

\author{
R. A. Beaumont \\ University of Washington \\ Seattle, Washington 98105
}

\section{J. DugundII}

Department of Mathematics

University of Southern California

Los Angeles, California 90007

D. Gilbarg and J. Milgram

Stanford University

Stanford, California 94305

\section{ASSOCIATE EDITORS}
E. F. BECKENBACH
B. H. NeumanN
F. WoLF
K. YoshidA

\section{SUPPORTING INSTITUTIONS}

\author{
UNIVERSITY OF BRITISH COLUMBIA \\ CALIFORNIA INSTITUTE OF TECHNOLOGY \\ UNIVERSITY OF CALIFORNIA \\ MONTANA STATE UNIVERSITY \\ UNIVERSITY OF NEVADA \\ NEW MEXICO STATE UNIVERSITY \\ OREGON STATE UNIVERSITY \\ UNIVERSITY OF OREGON \\ OSAKA UNIVERSITY
}

\author{
UNIVERSITY OF SOUTHERN CALIFORNIA \\ STANFORD UNIVERSITY \\ UNIVERSITY OF TOKYO \\ UNIVERSITY OF UTAH \\ WASHINGTON STATE UNIVERSITY \\ UNIVERSITY OF WASHINGTON \\ AMERICAN MATHEMATICAL SOCIETY
}

The Supporting Institutions listed above contribute to the cost of publication of this Journal, but they are not owners or publishers and have no responsibility for its contents or policies.

Mathematical papers intended for publication in the Pacific Journal of Mathematics should be in typed form or offset-reproduced (not dittoed), double spaced with large margins. Underline Greek letters in red, German in green, and script in blue. The first paragraph or two must be capable of being used separately as a synopsis of the entire paper. Items of the bibliography should not be cited there unless absolutely necessary, in which case they must be identified by author and Journal, rather than by item number. Manuscripts, in duplicate, may be sent to any one of the four editors. Please classify according to the scheme of Math. Reviews, Index to Vol. 39. All other communications should be addressed to the managing editor, or Elaine Barth, University of California, Los Angeles, California, 90024.

100 reprints are provided free for each article, only if page charges have been substantially paid. Additional copies may be obtained at cost in multiples of 50.

The Pacific Journal of Mathematics is issued monthly as of January 1966. Regular subscription rate: $\$ 72.00$ a year (6 Vols., 12 issues). Special rate: $\$ 36.00$ a year to individual members of supporting institutions.

Subscriptions, orders for back numbers, and changes of address should be sent to Pacific Journal of Mathematics, 103 Highland Boulevard, Berkeley, California, 94708.

PUBLISHED BY PACIFIC JOURNAL OF MATHEMATICS, A NON-PROFIT CORPORATION Printed at Jerusalem Academic Press, POB 2390, Jerusalem, Israel.

\section{Copyright (C) 1975 Pacific Journal of Mathematics All Rights Reserved}




\section{Pacific Journal of Mathematics

Vol. 60, No. $2 \quad$ October, 1975

Waleed A. Al-Salam and A. Verma, A fractional Leibniz q-formula ........... 1

Robert A. Bekes, Algebraically irreducible representations of $L_{1}(G) \ldots \ldots \ldots \ldots 11$

Thomas Theodore Bowman, Construction functors for topological

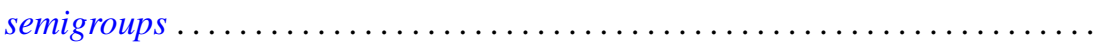

Stephen LaVern Campbell, Operator-valued inner functions analytic on the

closed disc. II .........................................

Leonard Eliezer Dor and Edward Wilfred Odell, Jr., Monotone bases in $L_{p} \ldots \ldots$.

Yukiyoshi Ebihara, Mitsuhiro Nakao and Tokumori Nanbu, On the existence of

global classical solution of initial-boundary value problem for

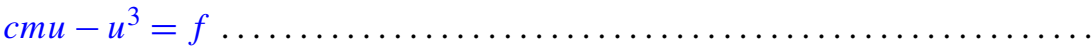

Y. Gordon, Unconditional Schauder decompositions of normed ideals of

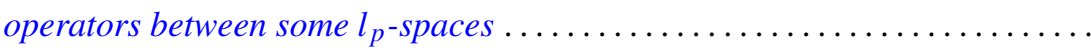

Gary Grefsrud, Oscillatory properties of solutions of certain nth order functional

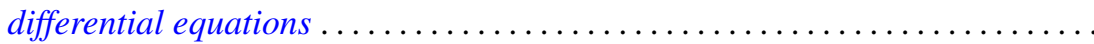

Irvin Roy Hentzel, Generalized right alternative rings ...................

Zensiro Goseki and Thomas Benny Rushing, Embeddings of shape classes of compacta in the trivial range .................................

Emil Grosswald, Brownian motion and sets of multiplicity . .

Donald LaTorre, A construction of the idempotent-separating congruences on a

bisimple orthodox semigroup .

Pjek-Hwee Lee, On subrings of rings with involution ...

Marvin David Marcus and H. Minc, On two theorems of Frobenius ...

Michael Douglas Miller, On the lattice of normal subgroups of a direct

product. .

Grattan Patrick Murphy, A metric basis characterization of Euclidean space

Roy Martin Rakestraw, A representation theorem for real convex functions ....

Louis Jackson Ratliff, Jr., On Rees localities and $H_{i}$-local rings ...

Simeon Reich, Fixed point iterations of nonexpansive mapping . .

Domenico Rosa, $B$-complete and $B_{r}$-complete topological algebras ...

Walter Roth, Uniform approximation by elements of a cone of real-valued

functions ....

Helmut R. Salzmann, Homogene kompakte projektive Ebenen

Jerrold Norman Siegel, On a space between $B H$ and $B_{\infty} \ldots$

235

Robert C. Sine, On local uniform mean convergence for Markov operators

James D. Stafney, Set approximation by lemniscates and the spectrum of an

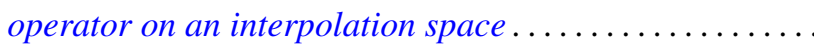

Árpád Száz, Convolution multipliers and distributions .......

Kalathoor Varadarajan, Span and stably trivial bundles ..........

Robert Breckenridge Warfield, Jr., Countably generated modules over

commutative Artinian rings....................... 